Correction

\title{
Correction: Laengner, M. L., et al. Trends in the Seaward Extent of Saltmarshes across Europe from Long-Term Satellite Data. Remote Sensing 2019, 11,1653
}

\author{
Marieke Liesa Laengner ${ }^{1, *}$, Koen Siteur ${ }^{1,2,3}$ and Daphne van der Wal ${ }^{1,4}$ \\ 1 NIOZ Royal Netherlands Institute for Sea Research, Department of Estuarine and Delta Systems, \\ Utrecht University, P.O. Box 140, 4400 AC Yerseke, The Netherlands; koen.siteur@nioz.nl (K.S.); \\ Daphne.van.der.Wal@nioz.nl (D.v.d.W.) \\ 2 Shanghai Key Laboratory for Urban Ecological Processes and Eco-Restoration, School of Ecological and \\ Environmental Science, East China Normal University, Shanghai 200241, China \\ 3 Center of Global Change and Ecological Forecasting, School of Ecological and Environmental Science, \\ East China Normal University, Shanghai 200241, China \\ 4 Faculty of Geo-Information Science and Earth Observation (ITC), University of Twente, P.O. Box 217, \\ 7500 AE Enschede, The Netherlands \\ * Correspondence: marieke.laengner@nioz.nl; Tel.: +31-(0)113-577-457
}

Received: 7 January 2020; Accepted: 9 January 2020; Published: 17 January 2020

In this paper [1], the authors have detected an error in the script that is provided as a template to apply the saltmarsh classification. One of the functions of the script is to calculate the size $\left(\mathrm{km}^{2}\right)$ of a study site, based on the number of total pixels in each study area. This method is applied to 125 study sites across Europe. The error that occurred is that instead of counting the pixels, the authors summed up all the values of the pixels (i.e., saltmarsh $=1$, mudflat $=2$, water $=3$ ) in each study area, leading to an overestimation of the total area of each site.

The authors wish to make the following corrections to this paper [1]:

1. Changes in the main body paragraphs

In Section 2.5, Thematic Classification Performance, on page 7 the authors wish to replace the link to the Google Earth Engine script:

https://code.earthengine.google.com/5941f820b68349f149f81786a8a9bb4a

with this link:

https://code.earthengine.google.com/81b0eca8b4c14d3083660ac3c06c2e46.

In Section 2.7, Method Application, on page 7 the authors wish to change the sentence:

"From this global data set, we included all European saltmarsh sites (systems with saltmarsh and mudflat and/or water) larger than $20 \mathrm{~km}^{2} . "$

To this sentence:

"From this global data set, we included all European saltmarsh sites (systems with saltmarsh and mudflat and/or water) larger than $20 \mathrm{~km}^{2}$, as well as 16 sites between $5 \mathrm{~km}^{2}$ and $20 \mathrm{~km}^{2}$,"

The threshold of the minimum size of the study site of $20 \mathrm{~km}^{2}$ does no longer hold as the size of the study site was overestimated before as explained above.

2. Change of figures

The authors wish to update Figure A3 with the correct size of each study site by replacing the old figure: 


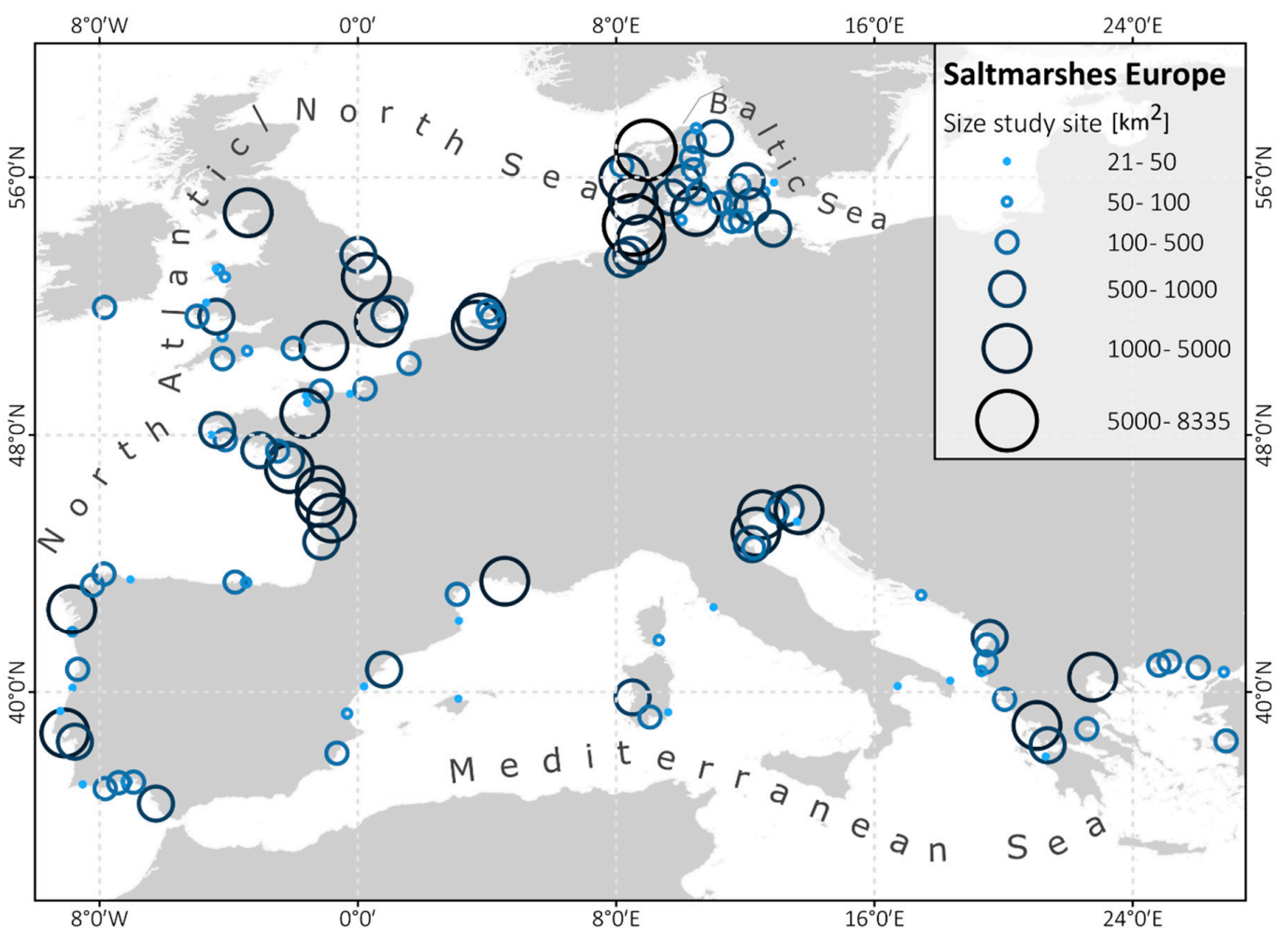

with the new figure:

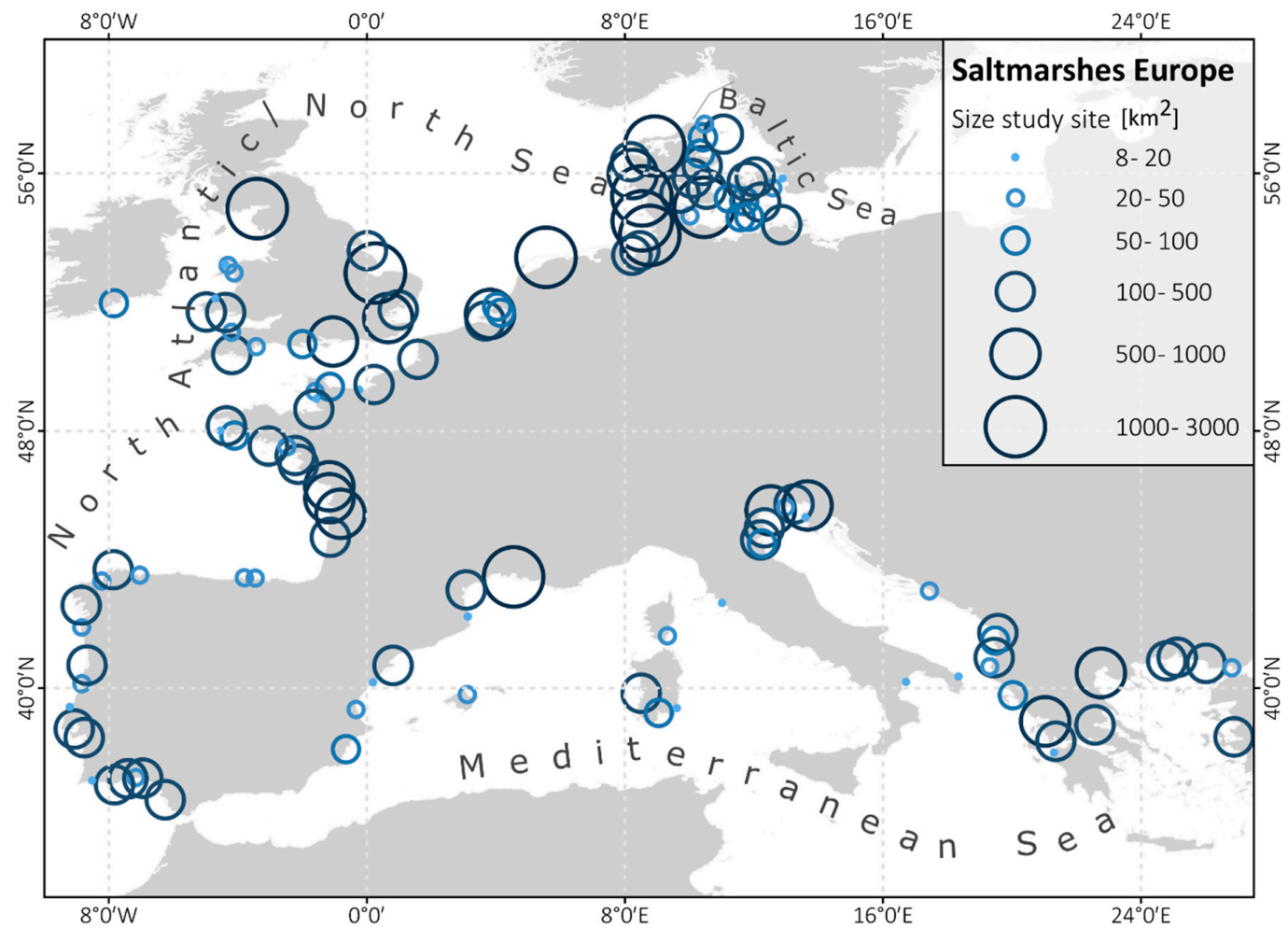


3. Change in the Appendix

In the Appendix a link is provided to the Google Earth Engine (GEE) script that is provided as a template to apply the saltmarsh classification. In order to refer to the corrected GEE-script the authors wish to replace this link:

https://code.earthengine.google.com/1528b64867f7286dcea7028f65a356c6

with this link:

https://code.earthengine.google.com/0b40364f8ca77ea6bf1fbdc4d717480d.

4. Changes in the Supplementary Materials

In the Supplementary Materials, the authors provide a list with the yearly habitat changes of the European saltmarshes; the third column of this list shows the total size of each study site $\left(\mathrm{km}^{2}\right)$. The authors wish to update this column with the correct size of each study site.

The errors and the consequential changes listed here do not affect any of the main results or the conclusions in this paper. The authors would like to apologize for any inconvenience caused to the readers by these changes.

\section{Reference}

1. Laengner, M.L.; Siteur, K.; van der Wal, D. Trends in the Seaward Extent of Saltmarshes across Europe from Long-Term Satellite Data. Remote Sens. 2019, 11, 1653. [CrossRef] 